\title{
B2K: the polarization-sensitive BOOMERanG experiment
}

\author{
S. Masi ${ }^{1}$, P.A.R. Ade ${ }^{2}$, J.J. Bock ${ }^{3}$, A. Boscaleri ${ }^{4}$, P. de Bernardis ${ }^{1}$, G. De Troia ${ }^{1}$, \\ G. Di Stefano ${ }^{5}$, V.V. Hristov ${ }^{6}$, A. Iacoangeli ${ }^{1}$, W.C. Jones ${ }^{6}$, T. Kisner ${ }^{7}$, \\ A.E. Lange ${ }^{6}$, P.D. Mauskopf ${ }^{2}$, C. Mac Tavish ${ }^{8}$, T. Montroy ${ }^{7}$, C.B. Netterfield ${ }^{8}$, \\ E. Pascale ${ }^{4}$, F. Piacentini ${ }^{1}$, F. Pongetti ${ }^{5}$, G. Romeo ${ }^{5}$, J.E. Ruhl ${ }^{7}$, E. Torbet ${ }^{7}$, J. Watt ${ }^{8}$ \\ ${ }^{1}$ Dipartimento di Fisica, Universitá La Sapienza, Roma, P.le A. Moro, 2, 00185, Italy. ${ }^{2}$ Dept. of Physics and \\ Astronomy, Cardiff University, Cardiff CF24 3YB, Wales, UK. ${ }^{3}$ Jet Propulsion Laboratory, Pasadena, CA, USA. \\ ${ }^{4}$ IROE-CNR, Firenze, Italy. ${ }^{5}$ Istituto Nazionale di Geofisica, Roma, Italy. ${ }^{6}$ California Institute of Technology, \\ Pasadena, CA, USA. ${ }^{7}$ Dept. of Physics, Univ. of California, Santa Barbara, CA, USA. ${ }^{8}$ Depts. of Physics and \\ Astronomy, University of Toronto, Canada.
}

\begin{abstract}
We describe the new BOOMERanG payload, which is being prepared for a new circum-antarctic flight, with the aim to detect the linear polarization of the Cosmic Microwave Background (CMB). In addition to polarization capabilities, obtained by means of special bolometers, the instrument has been improved in the attitude reconstruction system and in the calibration system.
\end{abstract}

\section{INTRODUCTION}

The BOOMERanG payload [1], [2], [3] has been flown in 1997 and 1998/99 and has produced high resolution maps of the microwave sky at $90,150,240,410 \mathrm{GHz}$ [4], [5]. The angular power spectrum of the maps features peaks at multipoles $\ell \sim 210,540,840$, [5], [6] as expected in all cosmological models with phase-coherent pressure waves in the photon-baryon fluid at recombination. These are expected in adiabatic models of structure formation, where fluctuations are generated in the inflation process. Independent evidence for these features comes from the DASI [7] and MAXIMA [8] experiments.

One strong prediction of this scenario is that the CMB should have a small level of linear polarization. In fact, in Thomson scattering, any quadrupole anisotropy in the incoming photons results in a degree of linear polarization in the scattered photons [9]. The main terms of the local anisotropy due to density (scalar) fluctuations are monopole and dipole, while the quadrupole term is much smaller: for this reason the expected polarization is quite weak [12], [10]. It is possible to compute the power spectra of the polarization expected for the same cosmological scenario best fitting the current anisotropy data. This scalar component of the CMB polarization is expected to be curl-free, and is called the EE component of the polarization. Moreover, in the inflationary scenario, tensor fluctuations (gravitational waves) are generated in the very early Universe, thus producing a different pattern of polarization in the CMB. These fluctuations introduce both curl-free and curl (labeled BB) components in the polarization pattern. There are thus several good reasons to search for such small polarization signature in the CMB sky. A cross correlation spectrum between temperature and scalar polarization (labeled TE) is expected as well (see e.g. [11]), with amplitude larger than the pure scalar and tensor polarization spectra. This is in the range of sensitivity of the MAP satellite, and will probably not require the development of a dedicated experiment. Typical levels of the rms polarization fluctuations in a $0.5^{\circ}$ beam are $\sim 1 \mu K$ for the $\mathbf{E E}$ component and $\sim 0.2 \mu K$ for the $\mathbf{B B}$ component. Compared to the temperature fluctuations (of the order of $70 \mu \mathrm{K} \mathrm{rms}$ in the same beam), these numbers are definitely challenging. Changing the cosmological parameters within the ranges consistent with the BOOMERanG measurements of the anisotropy does not change much the resulting polarization. The polarization signal is very small with respect to the CMB anisotropy itself, and even smaller with respect to many instrumental and astrophysical contaminants. Despite very intense and long lasting efforts [13], [14], [15], [16], [17], [18], [19], [21], [20], CMB polarization has not been detected yet. The best upper limits for the polarization power spectrum are still of the order of 5-10 $\mu \mathrm{K}$. 


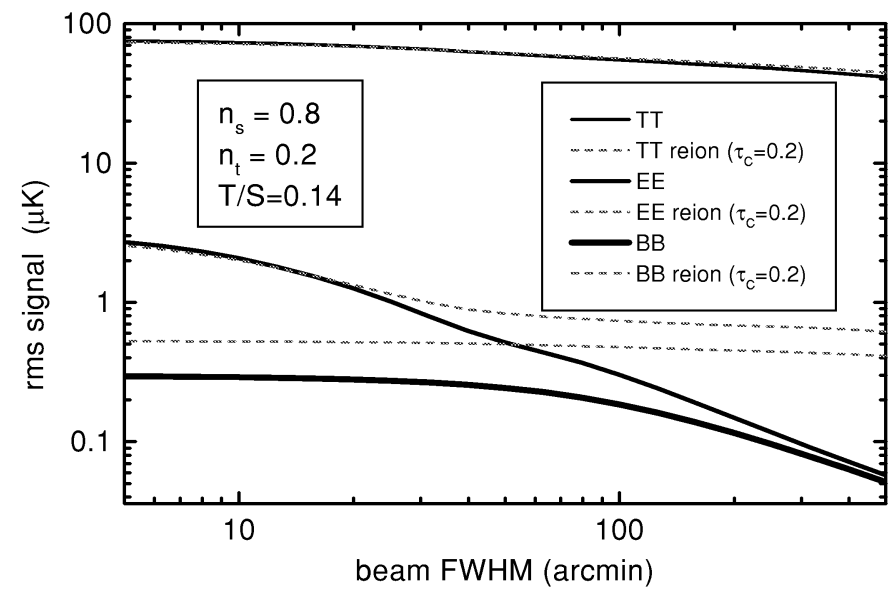

FIGURE 1. rms signal detected by a polarization experiment as a function of the beam FWHM. The temperature anisotropy (TT), gradient polarization (EE) and curl polarization (BB) signals are plotted. A model with a significant tensor component has been selected, for illustration purposes.

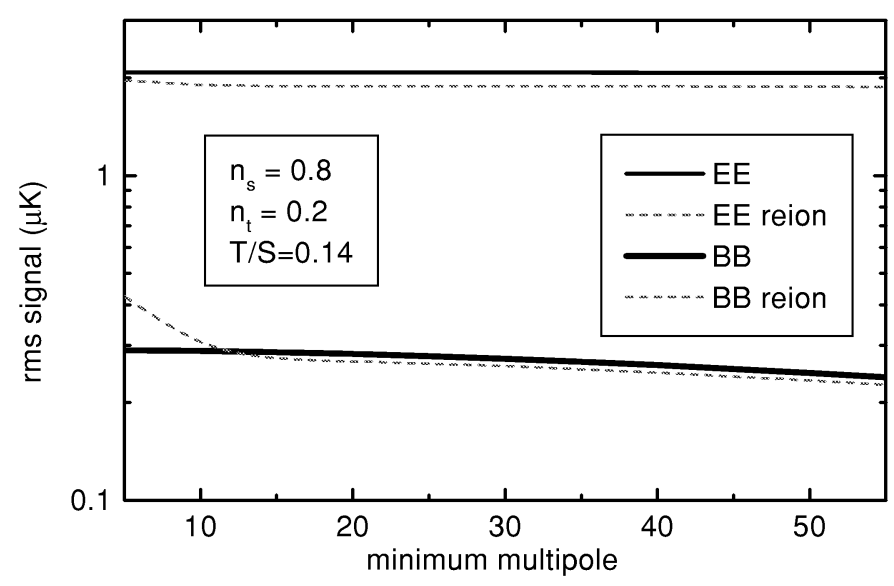

FIGURE 2. rms signal detected by a polarization experiment as a function of the minimum multipole to which the survey is sensitive. This is if the order of $\pi / \theta$, where $\theta$ is the maximum size of the survey.

We have a quite detailed idea of the signal we expect in the standard scenario. The angular power spectrum can be computed using CMBFAST, and the rms signal expected for a polarimeter covering a given multipoles range can be computed by integrating the power spectra. In fig. 1 we show how the detected rms signal depends on the angular resolution of the polarimeter, while in fig. 2 we show how the rms signal depends on the minimum multipole to which the polarimeter is sensitive. This, in turn, depends on the area of the sky survey (a size of the survey of $\theta$ allows detection of multipoles $\ell>\pi / \theta$ ). It is evident that, for a polarization experiment oriented to a first detection of $\mathbf{E E}$, a good angular resolution $\sim 1^{o}$ to $\sim 10^{\prime}$ should be preferred, while the size of the survey can be quite limited. The detection of the reionization signal and the measurement of $\tau_{C}$ would require, instead, a very wide survey. 


\section{EXPERIMENTAL APPROACH}

The experimental approach to the problem is to find an instrument setup able to extract the small CMB polarization signal in the presence of overwhelming CMB anisotropy, astrophysical and instrumental foregrounds.

For this purpose most polarimeters use a modulating analyzer to extract, by means of synchronous demodulation techniques, the small polarized component. Rotating wire grids, half wave plates, K-mirrors, Faraday rotators, Fresnel rombs, have been used or proposed as polarization analyzers (see e.g. [22]). All these techniques suffer for the need of long integration time: one needs to point to the same sky pixel during many cycles of the analyzer. It is thus difficult to produce extended maps of the $\mathrm{CMB}$ polarization.

In BOOMERanG we use the sky scan to modulate the signal. We want to map the two orthogonal components of the linear polarization with two separate bolometers, $B 1$ and $B 2$, each sensitive to one polarization direction, and combine the two signals to retreive the linear polarization Stokes parameter Q. The U parameter can be measured as well by means of an identical device rotated by $45^{\circ}$. (see fig.4). Polarization sensitive bolometers (PSB) of this kind have been prepared for the coming flight of BOOMERanG and for the High Frequency Instrument of the Planck satellite [23].

Very little is known about the polarization of CMB foregrounds. Synchrotron radiation is strongly polarized, and microwave emission from spinning dust grains is also expected to be significantly polarized. For this reason, the best spectral region for this search is in the $150 \mathrm{GHz}$ range, where thermal emission from interstellar dust (ISD) is low enough [24], and the two above mentioned polarized foregrounds are not an issue. If we assume a conservative 5\% polarization for the dust (see e.g. [25]), the resulting polarized signal at $150 \mathrm{GHz}$ is lower than the CMB polarization to be detected (see fig.3).

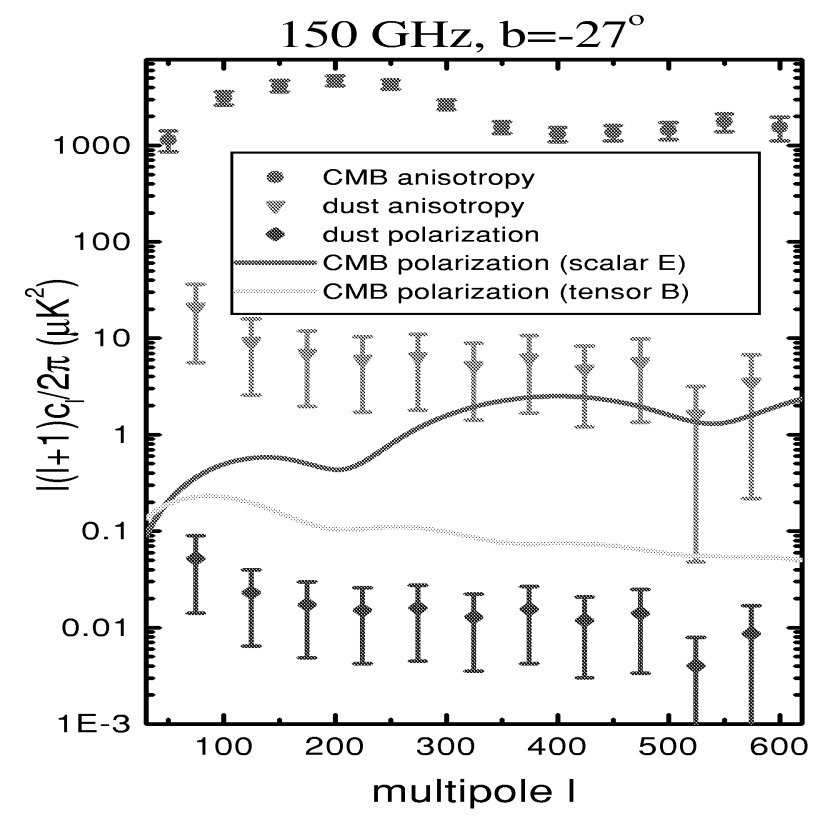

FIGURE 3. Comparison between anisotropy and polarization of the CMB and anisotropy and polarization of interstellar dust in high latitude cirrus clouds. The anisotropy measurements are from the BOOMERanG experiment at $150 \mathrm{GHz}$. The CMB polarization power spectra are computed assuming the best fit parameters obtained fitting the anisotropy power spectrum; the interstellar dust anisotropy has been extrapolated to $150 \mathrm{GHz}$ using the measurements at $410 \mathrm{GHz}$, and the polarization of interstellar dust has been estimated simply assuming a conservative $5 \%$ of the anisotropy. 


\section{B2K}

With the BOOMERanG payload, recovered and refurbished after the 1998 flight, signal modulation is obtained by scanning the sky at a constant rate $v$. Polarization signals are detected at a frequency $f$ far from the $1 / \mathrm{f}$ knee of the noise and far from the effects of instrumental drifts. This sky scan approach is similar to the anisotropy measurements performed with BOOMERanG in its long duration flight in 1998. In fact $f=v \ell / \pi$; with $\ell=300-1000$ and $v=1^{\circ} / \mathrm{s}$ we get $f=1.7-5.5 \mathrm{~Hz}$, well inside the useful bandwidth of the $0.3 \mathrm{~K}$ bolometers used in the BOOMERanG instrument.

In BOOMERanG an off-axis telescope close to the Dragone configuration [26] has been used. We have analyzed in detail its polarization properties on a wide region of the focal plane. The telescope is an off-axis gregorian, with a $1.3 \mathrm{~m}$ diameter aluminum primary enclosed in a low emissivity cavity. Secondary and tertiary are inside the cryostat, and reimage the primary focal plane inside the dewar. The cold tertiary acts as the Lyot stop of the system, and is surrounded by a $2 \mathrm{~K}$ absorber, thus improving sidelobes rejection and significantly reducing the straylight on the detectors. The spurious polarization induced by the BOOMERanG off-axis telescope at the edge of the used focal plane is $\sim 10^{-4}$, well suited for this purpose.

Combining signals from two different detectors instead of using always the same detector to measure the two polarization components is a significant concern (see e.g. [27]). One has to demonstrate that this procedure does not introduce significant systematics. Spurious polarization induced by the optics or by the bolometers polarizes any unpolarized input signal. Since the spurious polarization is constant during the scans, only sky brightness gradients are a concern, while the constant instrumental and astrophysical backgrounds do not affect the measurements. Cross polarization induced in the optics or in the PSBs can be treated in a similar way. We have carried out detailed simulations to investigate the problem in a quantitative way. We find that, if the dominant unpolarized signal is CMB anisotropy, spurious polarization and unbalanced cross polarization up to a few $\%$ are acceptable . A careful pre-flight calibration is required, to measure the cross-polarization of the system to a few \% [28].

The B2K1 focal plane design was optimized for sensitivity per pixel and foreground discrimination. It consists of 8 close-packed corrugated feed elements arranged in 2 rows separated by an elevation of $0.5^{\circ}$ (see fig.4). Each row contains 4 elements separated in cross-elevation by $0.5^{\circ}$. In the upper row corrugated shaped horns feed 2-color photometers which operate at $245 \mathrm{GHz}$ and $345 \mathrm{GHz}$. Polarizing grids are placed on the front of photometer feed horns. The photometers use spider web bolometers similar to the ones used in B98. The horns in the lower row feed
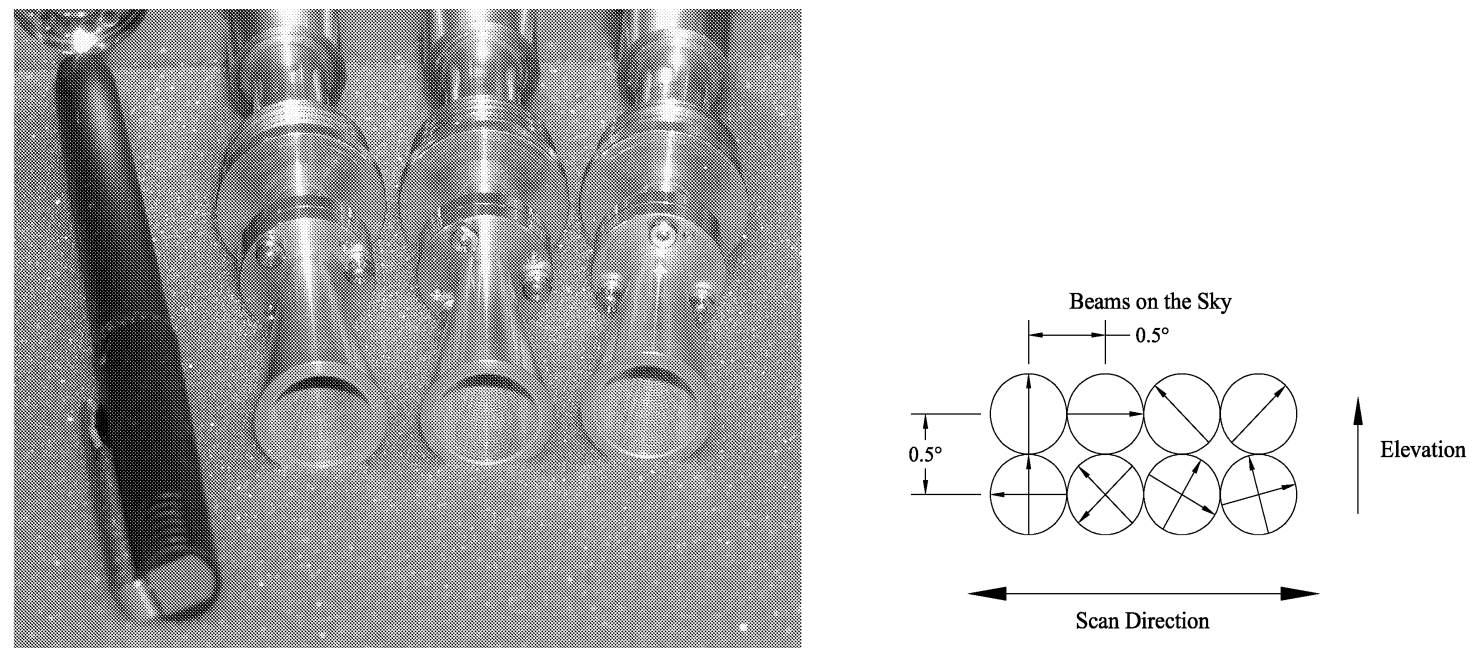

FIGURE 4. Left: Photo of three corrugated feedhorns used in the $150 \mathrm{GHz}$ channels of B2K. Right: sketch of the B2K focal plane. The upper row of feeds has grid polarizers at the entrance, and 2-color photometers at 245 and $345 \mathrm{GHz}$ at the output. The lower row of feeds has $150 \mathrm{GHz}$ polarization sensitive bolometers (PSBs) at the output.

pairs of PSB operating at $150 \mathrm{GHz}$. The PSB's consist of 2 square mesh bolometers placed $65 \mu \mathrm{m}$ apart in the same groove of a circular corrugated waveguide. Each bolometer is metallized along one direction and each element in the pair is sensitive to a different polarization (see fig.5). The PSBs provide an instantaneous measurement of the Stokes I and Q parameters. The relative rotation of each pixel allows the determination of the U parameter as well as I and Q. Each bolometer has an in-flight NET of $170 \mu K_{C M B} \sqrt{s}$. The instrumental sensitivity to temperature anisotropies will 


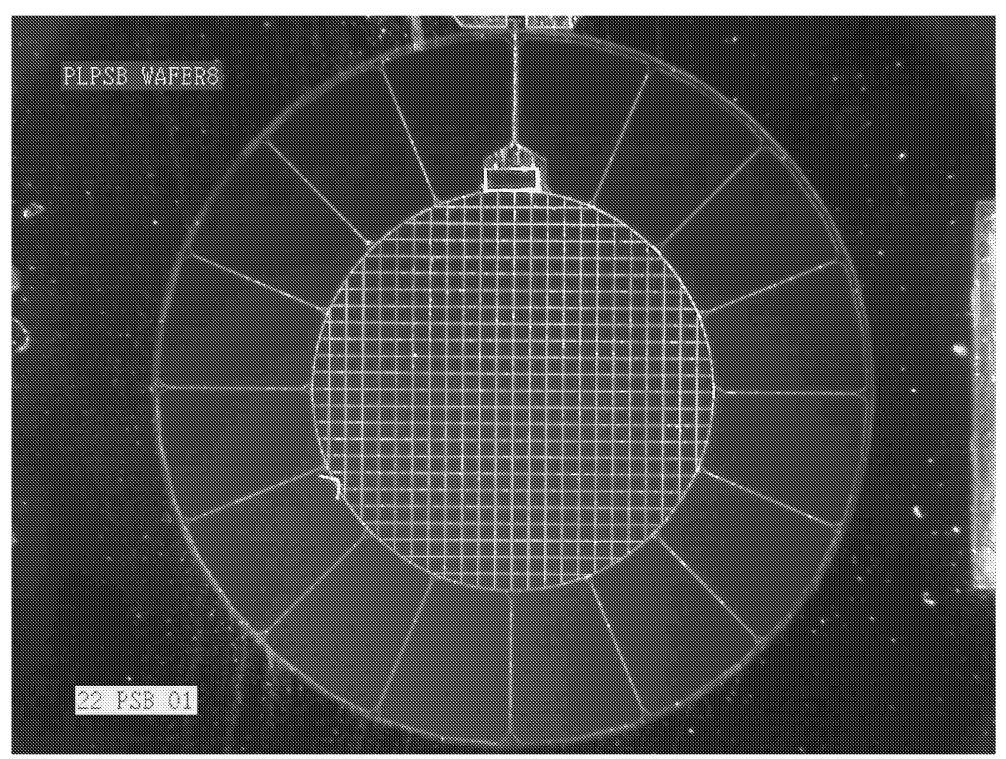

FIGURE 5. Photograph of a PSB used in the B2K focal plane. The two grids of the two separate bolometers are overlap in the picture. The grids have a diameter of $2.6 \mathrm{~mm}$ and are made out of $2 \mu \mathrm{m}$ thick $\mathrm{Si}_{3} \mathrm{~N}_{4}$ wires metallized only in one of the two orthogonal directions.

be of the order of $170 / \sqrt{8}=60 \mu K_{C M B} \sqrt{s}$, with a sensitivity to $\mathrm{Q}$ and $\mathrm{U}$ of $\sim 170 \times \sqrt{2} / \sqrt{4}=120 \mu K_{C M B} \sqrt{s}$.

The frequency bands are determined using metal mesh filters for the low pass and waveguide cut-offs for the high pass. The PSB's are expected to have a beam FWHM of 11 arcmin and the photometer channels have a 6 arcmin FWHM. The feed elements are placed as close as mechanically possible to optimize the overlap of maps made by different channels. This is especially critical for the photometers, since Stokes Q and U can be determined only by differencing maps made by different photometers.

\section{POLARIMETER CALIBRATION}

We have seen above how important it is to characterize the polarization properties of CMB polarimeters. The pre-flight calibration of a polarimeter is more difficult than the usual photometer calibration. In particular it is very important to study the co-polar and cross-polar response (beam and integral) of the polarimeter. We have developed a polarized, far field, sine-modulated source filling the beam of the instrument. This will allow us to carry out a through polarization characterization of all the detectors in the instrument. A 10' diameter beam is defined by a stop in the focus of a 1.3 $\mathrm{m}$ off-axis paraboloid (the spare of the BOOMERanG mirror). Placing a small size source in the center of the focal plane we limit the spurious polarization induced by the off-axis mirror to $<10^{-4}$. Behind the stop there are two slant wire grid polarizers (P1 and P2), and a 77K black-body source. Rotating P2 at constant speed we modulate the signal, producing a sine wave modulation at twice the rotation frequency. Rotating P1 (in steps) we change the illuminator from co-polar to cross-polar (and all intermediate directions). With this source we plan to measure the co-polar and cross-polar response for each detector in the BOOMERanG focal plane with better than $1 \%$ accuracy.

\section{THE NEW ACS SENSORS}

In addition to the Attitude Control System (ACS) sensors used in the previous flight (see [30],[2]) we are developing for the new payload two daytime star trackers. This kind of imaging sensors -ccd based in our case- gives full attitude reconstruction, with an accuracy that can be as good as few arc-seconds, if at least two stars are framed at one time. To run the star tracker in daytime, we have to deal with the diffused sky background $[31,32,33]$ that, even at balloon altitude, is not negligible. As a general rule, this background is proportional to the atmospheric pressure [34] and, 
hence, it is few thousandths than at sea level. In order to be able to detect a faint star, we must require that the CCD sensor will not saturate and that it has enough dynamic range to separate the star signals from the bright background. One of our star trackers is based on the Kodak KAF1401 sensor, featuring $70 \mathrm{~dB}$ of dynamic range and a well capacity of $45 \mathrm{ke}^{-}$. We found that, to be able to have at least one star framed at one time, we need to detect magnitude 4 stars, with a field of view (FOV) of 8 degrees. Since the KAF sensor has a sensitive area of $8.9 \times 7.04 \mathrm{~mm}^{2}$ we need to use a $50 \mathrm{~mm}$ lens. A 4 th magnitude star focused on 9 pixels at $f=0.8$ gives a signal that is roughly 30 times fainter than the background. This can be detected by taking advantage of the wide dynamic range of the sensor, that allows us to use a 14 bit ADC (see [34]). This sensor will not be moving with respect to the payload during the scans. The wide FOV assures detection of the same stars during the scans.
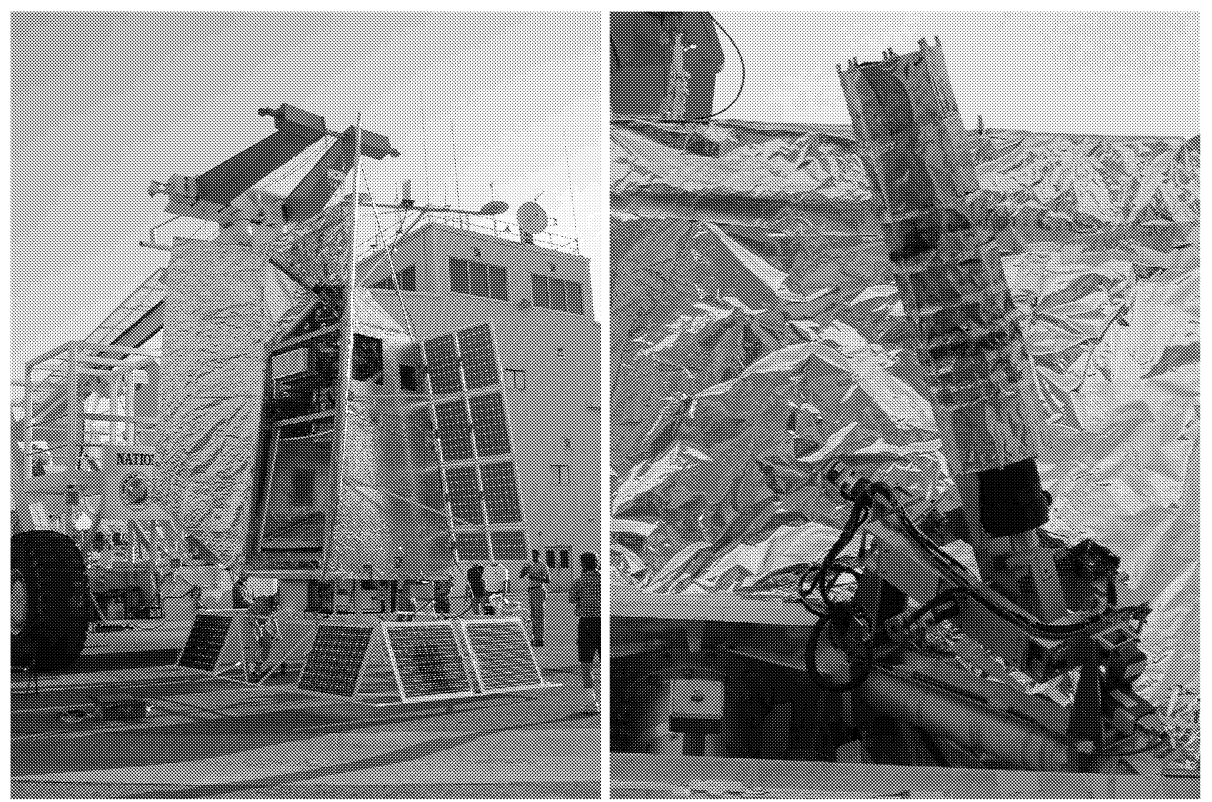

FIGURE 6. Left: The integrated B2K payload (telescope earth shield removed). Right: The tracking star camera mounted on the B2K payload.

The other camera (see fig.6) will have a smaller FOV (about $0.5^{\circ}$ ) and will track the reference star during the scans. The star position will be recovered combining the position of the star on the CCD array and the position angles of the camera with respect to the gondola, measured by two 16 bit absolute encoders.

The combined use of these two new sensors will allow sub-arcmin attitude reconstruction of the payload.

\section{CONCLUSIONS}

The BOOMERanG payload has been refurbished and improved in critical areas like calibration and pointing reconstruction. The addition of PSBs in the focal plane allows the search for the elusive CMB polarization. The system is scheduled to fly from Antarctica at the end of 2002.

\section{ACKNOWLEDGMENTS}

This activity is being supported by Universita' di Roma La Sapienza, PNRA and ASI in Italy, NSF and NASA in the USA, PPARC in UK and CIAR and NSERC in Canada.

\section{REFERENCES}

1. Masi S., et al., in "3K cosmology", AIP Conf. Proc. 476, 237, (1999); astro-ph/9911520 
2. Piacentini F., et al. 2001, astro-ph//0105148 (Ap.J. in press).

3. Crill B.P., et al., in preparation (2002). See also Crill B.P., Ph.D. thesis, Caltech, 2000.

4. de Bernardis, P. et al. 2000, Nature, 404, 955

5. Netterfield C.B., et al. (2001), astro-ph/0104460

6. de Bernardis, P., et al., 2002, Ap.J. in press; astro-ph/0105296

7. Halverson N.W. et al. 2001, astro-ph/0104489

8. Lee A.T. et al. 2001, astro-ph/0104459

9. Rees M., Ap.J., 153, L1, (1968)

10. Hu W., White M., New A. 2, 323, (1997)

11. Kaminokowsky M., Kosowsky A., Ann.Rev.Nucl.Part.Sci. 49, 77-123, (1999)

12. Kaiser N., MNRAS, 202, 1169, (1983)

13. Caderni N., Phys.Rev.D., 17, 1901, (1978); Phys.Rev.D., 17, 1908, (1978)

14. Nanos G.P., Ap.J., 232, 241, (1979)

15. Lubin P. and Smoot G., Ap.J., 245, 1, (1981)

16. Partridge B., et al., Nature, 331, 146, (1988)

17. Wollack et al., Ap.J., 476, 440, (1997)

18. Netterfield B., et al., Ap.J., 474, 47, (1997)

19. Hedman M.M. et al., Ap.J., 548, L111, (2001); astro-ph/0010592

20. Subrahmanyan R., et al., MNRAS, 315, 808 (2001)

21. Keating B., et al., 2001, "A Limit on the Large Angular Scale Polarization of the Cosmic Microwave Background", astro-ph/0107013

22. Pisano G., New Astron. Reviews, 43, 329, (1999)

23. Bock J., et al., in "2K1BC Workshop : Experimental Cosmology at Millimetic Wavelenghts" de Petris and Gervasi editors, AIP.

24. Masi S., et al., Ap.J., 553, L93, (2001A)

25. Tegmark M., et al., Ap.J., 530, 133, (2000)

26. Dragone M., IEEE Trans. AP-30, 331, (1982); IEEE Trans.AP-22, 472, (1974)

27. Carretti E., et al., 2001, "Limits Due to Instrumental Polarisation in CMB Experiments at Microwave Wavelengths", astro-ph/0103318

28. Masi S., et al., 2001, "Scanning polarimeters for measurements of CMB polarization", in "2K1BC Workshop : Experimental Cosmology at Millimetic Wavelenghts" de Petris and Gervasi editors, AIP.

29. Philhour B., et al., 2001, "The Polatron: A Millimeter-Wave Cosmic Microwave Background Polarimeter for the OVRO $5.5 \mathrm{~m}$ Telescope", astro-ph/0106543

30. Romeo G., et al., 2001, "Three Sun Sensors for Stratospheric Balloon Payloads", in "2K1BC Workshop : Experimental Cosmology at Millimetic Wavelenghts" de Petris and Gervasi editors, AIP.

31. Yves André "Conception et developpement de senseurs stellaires diurnes embarques a bord de nacelles de ballons stratospheriques" P.h.D thesis

32. E. Rossi et Al., "The in-flight performance of the zebra day-time star sensor" Advance in space research, Vol.13, No.2, 1993, Pag. 159-163

33. E. Pascale, C. MacTavish, "Daytime Sky Brightness at Stratospheric Balloon Altitude", 2002, In preparation.

34. E. Pascale, A. Boscaleri, "A daytime bore-sight star tracker for balloon experiments", 2002, In preparation 